\title{
Associação entre consumo alimentar, atividade física, fatores socioeconômicos e percentual de gordura corporal em escolares
}

\author{
Association between dietary intake, physical activity, socioeconomic \\ factors and body fat percentage among schoolchildren
}

Gisele Liliam D’Avila ${ }^{1}$

Diego Augusto Santos Silva ${ }^{2}$

Francisco de Assis Guedes de Vasconcelos ${ }^{3}$

${ }^{1}$ Programa de PósGraduação em Nutrição, Centro de Ciências da Saúde, Universidade Federal de Santa Catarina (UFSC). Campus Universitário, Trindade. 88.040-900 Florianópolis SC Brasil. gisele_liliam@hotmail.com

${ }^{2}$ Departamento de Educação Física, Centro de Desportos, UFSC Florianópolis SC Brasil.

${ }^{3}$ Departamento de Nutrição, Centro de Ciências da Saúde, UFSC. Florianópolis SC Brasil.

\begin{abstract}
The scope of this article was to assess the association between dietary intake, physical activity and socioeconomic factors associated with body fat percentage in 7 to 14-year-old schoolchildren. It is a cross-sectional study with a probability sample of 2,481 students from public and private schools in Florianópolis, Santa Catarina, Brazil. Body fat percentage was investigated by measuring triceps and subscapular skin folds. Poisson regression was performed to test the association between excess body fat and independent variables, estimating prevalence ratios and 95\% confidence intervals. The prevalence of excess body fat was 23.9\%, though there was no significant difference between sex $(p=0.359)$ and age ( $p$ $=0.202$ ). Excess body fat was associated with different factors in 11 to 14-year-old schoolchildren, namely eating less than three meals a day $(\mathrm{OR}=$ 1.62, CI: 1.38 to 1.91) and consumption of highrisk food more than 3 times a day $(O R=0.61$ CI: 0.47 to 0.79). No significant difference was observed with physical activity. The high prevalence of excess body fat detected reveals the need for fostering the adoption of healthier behavioral practices (in relation to nutrition and physical activity) by schoolchildren.

Key words Dietary intake, Schoolchildren, Body composition, Physical activity, Socioeconomic factors
\end{abstract}

Resumo O objetivo do artigo foi verificar a associação entre consumo alimentar, atividade física, fatores socioeconômicos e percentual de gordura corporal em escolares de 7 a 14 anos de idade. Estudo transversal realizado com amostra probabilistica de 2481 escolares matriculados em escolas públicas e privadas de Florianópolis, Santa Catarina, Brasil. O percentual de gordura corporal foi investigado por meio das dobras cutâneas do tríceps e subescapular. Foram realizadas análises brutas e ajustadas por meio da regressão de Poisson, estimando-se razões de prevalência e intervalos de confiança de $95 \%$. A prevalência do excesso de gordura corporal foi de 23,9\%, não apresentando diferença significativa entre sexo $(p=0,359) e$ idade ( $p=0,202)$. A gordura corporal associou-se a diferentes fatores nos escolares de 11 a 14 anos: consumir menos que 3 refeições ao dia $(R P=1,62$; IC: $1,38-1,91)$ e alimento de risco mais que 3 vezes ao dia $(R P=0,61$; IC: 0,47-0,79). Não foi observada diferença significativa com atividade física. A elevada prevalência de excesso de gordura corporal verificada evidencia a necessidade de estímulo à adoção de práticas comportamentais mais saudáveis (em relação à alimentação e à atividade fisica) pelos escolares.

Palavras-chave Consumo de alimentos, Escolares, Composição corporal, Atividade física, Fatores socioeconômicos 


\section{Introdução}

Estudos sobre a obesidade em escolares, diagnosticada a partir do Índice de Massa Corporal (IMC), têm apresentado distintas taxas de prevalência entre os países, tais como China $(3,3 \%)^{1}$, México $(14,7 \%)^{2}$, Paquistão $(7,5 \%)^{3}$ e Austrália $(6,6 \%)^{4}$.

No Brasil, as prevalências de obesidade em escolares de 7 a 14 anos de idade, baseadas no IMC, oscilam entre 5,5\% a 8,9\% $\%^{5-9}$. Pesquisa realizada em diferentes regiões do país, com escolares de 7 a 9 anos de idade, constatou prevalência de 7,8\% para obesidade 5 . Estudo realizado em Belém, Pará, com escolares de 6 a 19 anos de idade, identificou prevalência de obesidade de $8,9 \%{ }^{6}$. Em Niterói, Rio de Janeiro, observou-se uma prevalência de $7,7 \%$ de obesidade em escolares de 10 a 18 anos de idade 7 . No estado de Santa Catarina, pesquisas com escolares de 7 a 14 anos de idade encontraram valores de $5,5 \%$ a $6,1 \%$ de obesidade ${ }^{8-9}$.

A literatura tem evidenciado que entre os fatores de risco para o desenvolvimento da obesidade em escolares estariam as práticas alimentares inadequadas como o baixo consumo de frutas, legumes e verduras ${ }^{10}$; o alto consumo de doces, guloseimas, bebidas açucaradas e gorduras $^{10}$; o número reduzido de refeições diárias ${ }^{11} \mathrm{e}$ a omissão do café da manhã ${ }^{12}$. Alguns estudos sugerem ainda que fatores demográficos e socioeconômicos, como sexo, faixa etária, renda familiar, rede de ensino, escolaridade dos pais e fatores comportamentais como pouca atividade física estão associados à obesidade ${ }^{13-17}$.

O IMC tem sido frequentemente usado na avaliação da situação epidemiológica da obesidade dentro do contexto mundial ${ }^{1-9}$. No entanto, este índice apresenta importantes limitações metodológicas, especialmente no diagnóstico nutricional de crianças e adolescentes, uma vez que neste grupo etário o IMC está mais relacionado com o crescimento corporal, do que com mudanças relacionadas com a quantidade e distribuição da gordura corporal ${ }^{18}$. A literatura relata ainda outras limitações do uso do IMC em estudos epidemiológicos, tais como a diversidade de curvas de IMC utilizadas para diagnóstico da obesidade em adolescentes e o agrupamento de dados em diferentes faixas etárias, sem levar em consideração a maturação sexual ${ }^{19}$. Nesse sentido, outras medidas corporais têm sido utilizadas no diagnóstico da obesidade. A medição da gordura corporal por meio de dobras cutâneas é uma alternativa recomendada, porque este pro- cedimento antropométrico possibilita aferição tanto da quantidade como da distribuição corporal de tecido adiposo, significando, portanto, uma relação mais estreita com a obesidade ${ }^{20,21}$.

No Brasil, os estudos sobre prevalência e fatores associados à obesidade em escolares de 7 a 14 anos de idade têm tido como parâmetro predominante de diagnóstico o IMC ${ }^{8,9}$. Poucos estudos têm investigado a relação entre obesidade em crianças e adolescentes e seus fatores associados, tendo como base medidas antropométricas que possibilitem diagnósticos do percentual de gordura corporal ${ }^{14,16,17,22}$. Com isso, o objetivo do presente estudo foi verificar a associação entre consumo alimentar, atividade física, fatores socioeconômicos e percentual de gordura corporal em escolares de 7 a 14 anos de idade de Florianópolis, Santa Catarina, Brasil.

\section{Métodos}

Trata-se de estudo de caráter transversal vinculado à pesquisa mais abrangente financiada pelo Conselho Nacional de Desenvolvimento Científico e Tecnológico (CNPq), com amostra probabilística de escolares de 7 a 14 anos de idade de escolas públicas e privadas no município de Florianópolis, Santa Catarina. Detalhes dos procedimentos metodológicos foram descritos em estudo anterior ${ }^{23}$.

Para estimativa do tamanho da amostra utilizou-se uma prevalência de sobrepeso/obesidade esperada para 2012 de $38 \%$, com base em pesquisas anteriores realizadas com escolares de 7 a 14 anos de idade de Florianópolis ${ }^{8,24}$, baseadas no índice de massa corporal. Levando-se em conta o número total de escolares de 7 a 14 anos de idade $(\mathrm{n}=45247)$ do município de Florianópolis em 2010, erro amostral de 3,5 pontos percentuais (bicaudal) e um intervalo de confiança de 95\%, o tamanho de amostra necessário para a pesquisa seria de 727 crianças. Considerando um efeito de delineamento de 1.8, o tamanho de amostra necessário seria de 1309 crianças. Considerando a estratificação por faixa etária (7-10 anos e 11-14 anos), o tamanho de amostra foi duplicado, totalizando 2618 crianças a serem avaliadas. Acrescentando $10 \%$ a este valor por eventuais perdas ou recusas à pesquisa, o tamanho de amostra final foi de 2880 crianças.

O processo de amostragem foi realizado por conglomerados. As escolas foram divididas em estratos, de acordo com as regiões administrativas do município de Florianópolis (Centro, 
Continente, Norte, Leste e Sul) e o tipo de escola (Pública ou Privada). Em cada estrato foram selecionadas aleatoriamente as escolas a serem incluídas no estudo. A amostra incluiu 30 escolas (19 públicas e 11 privadas).

Foram incluídos no estudo os escolares que tiveram o Termo de Consentimento Livre e Esclarecido (TCLE) assinado pelos pais. Foram excluídos da pesquisa os escolares portadores de deficiência física que impossibilitasse a avaliação antropométrica e as adolescentes grávidas.

Uma vez que o presente estudo procurou testar as variáveis associadas com o excesso de gordura corporal, cálculos a posteriori foram realizados para estimar as mínimas diferenças detectáveis. Com base no tamanho da amostra disponível, na prevalência do desfecho e das variáveis independentes, poder de estudo de $80 \%$, nível de confiança de $95 \%$, desconto de $10 \%$ no tamanho de amostra pelo ajuste para fatores de confusão e efeito de delineamento de 1,5 para o desfecho, este estudo teria poder suficiente para detectar razões de prevalência de 0,67 a 0,85 como proteção e de 1,17 a 1,49 como fator de risco.

A coleta de dados foi realizada no período de setembro de 2012 a junho de 2013. A equipe responsável pela coleta foi composta por nutricionistas, educadores físicos, acadêmicos dos Cursos de Graduação em Nutrição e Educação Física e Pós-Graduação em Nutrição da Universidade Federal de Santa Catarina (UFSC).

A equipe de coleta recebeu treinamento para padronização das medidas antropométricas a fim de mensurar os erros intra e interavaliadores ${ }^{25}$. Para o cálculo do Erro Técnico de Medição (ETM) intra e interavaliador cada antropometrista aferiu as medidas antropométricas em dez escolares, para o erro intra-avaliador, foi considerado como erro aceitável o valor de até duas vezes a medida ETM do antropometrista de referência. Para determinar o erro interavaliador foi considerado como erro aceitável o valor de até três vezes a medida ETM do antropometrista de referência ${ }^{25}$.

A variável dependente, percentual de gordura corporal (\% de GC), foi construída com base nas medidas das dobras cutâneas triciptal (DCT) e subescapular (DCS). A partir da aferição destas dobras foi estimado o \% de GC pela equação preditiva de Lohman ${ }^{26}$. A equação de Lohman $^{26}$ é a seguinte: $\% \mathrm{GC}=1,35 \mathrm{x}(\mathrm{DCT}+\mathrm{DCS})-0,012 \mathrm{x}$ $(\mathrm{DCT}+\mathrm{DCS})^{2}$ - Intercepto. O intercepto, conhecido também como constante, varia de acordo com o sexo e a idade do jovem. Lohman ${ }^{26}$ sugeriu constantes para meninos e meninas nas idades de 7, 10, 13 e 16 anos. No presente estudo consideraram-se as constantes de Lohman ${ }^{26}$ para as idades de 7, 10 e 13 anos. Para as idades de 8, 9, 11, 12 e 14 anos consideraram-se as constantes sugeridas por Pires-Neto e Petroski ${ }^{27}$ que desenvolveram tais constantes para serem empregadas nessa equação de Lohman ${ }^{26}$ a partir de amostra de crianças e adolescentes brasileiros. Assim, as constantes empregadas na equação diferenciaram-se para meninos e meninas conforme a idade (Meninos: 7 anos $=3,4 ; 8$ anos $=3,7 ; 9$ anos $=$ 4,$1 ; 10$ anos $=4,4 ; 11$ anos $=4,7 ; 12$ anos $=5,0 ; 13$ anos $=5,4 ; 14$ anos $=5,7$, Meninas: 7 anos $=1,4$; 8 anos $=1,7 ; 9$ anos $=2,0 ; 10$ anos $=2,4 ; 11$ anos $=2,7 ; 12$ anos $=3,0 ; 13$ anos $=3,4 ; 14$ anos $=3,6$ ).

A coleta das medidas de espessura das dobras cutâneas foi realizada com adipômetro cientifico Lange ${ }^{\varpi}$ com escala de $1 \mathrm{~mm}$ em dois pontos anatômicos (tríceps e subescapular), segundo procedimentos padronizados por Lohman et al. ${ }^{28}$ As medidas foram realizadas do lado direito do avaliado, sendo feitas três da mesma dobra, de forma não consecutiva, adotando-se a media aritmética como medida final.

A variável dependente foi dicotomizada em: sem excesso de gordura corporal e com excesso de gordura corporal, com base na classificação sugerida por Williams et al. ${ }^{29}$. Por meio desta classificação foram considerados com excesso de gordura corporal aqueles sujeitos que apresentaram resultado de $\%$ de $\mathrm{GC} \geq 30$ para meninas e $\geq$ 25 para meninos.

As variáveis independentes foram rede de ensino (pública e privada); escolaridade dos pais (> 12 anos; 9 - 11 anos e $0-8$ anos); renda mensal familiar (em tercis de distribuição de reais); consumo de alimentos protetores (adequado e inadequado); consumo de alimentos de risco (adequado e inadequado); realização do café da manhã (não ou sim); número de refeições/dia (> 4 e $\leq 3$ ) e atividade física (numérica contínua).

Dados referentes à escolaridade dos pais e renda mensal familiar foram obtidos por meio de um questionário preenchido pelos pais, enviado juntamente com o TCLE. Os dados de identificação do escolar (nome, data de nascimento, sexo e rede de ensino) foram obtidos a partir da relação dos alunos pertencentes às turmas sorteadas, disponibilizadas pelas escolas participantes.

As informações de consumo de alimentos de proteção e de risco, realização do café da manhã, número de refeições, foram coletados por um Questionário de Alimentação do Dia Anterior (QUADA) versão 3, instrumento desenvolvido e validado por Assis et al. ${ }^{30}$. No QUADA, as refei- 
ções são ordenadas cronologicamente (café, lanche da manhã, almoço, lanche da tarde, jantar e lanche da noite). Cada refeição é ilustrada com 21 alimentos ou grupos de alimentos e pode gerar dados válidos para a avaliação do consumo de alimentos do dia anterior ${ }^{31}$. Na validação do QUADA, em média, os valores de sensibilidade e especificidade foram de $70,2 \%$ e $96,2 \%$ respectivamente ${ }^{30}$.

De acordo com os grupos de alimentos contidos no QUADA, consideraram-se alimentos protetores os pertencentes ao grupo das frutas, sucos naturais, vegetais folhosos, legumes, sopa de legumes e verduras. Como alimentos de risco os pertencentes ao grupo dos achocolatados, sucos artificiais, refrigerantes, doces, salgadinhos industrializados, batatas fritas e lanches rápidos ${ }^{32}$.

Seguindo as recomendações do Guia Alimentar para a População Brasileira ${ }^{32}$, o consumo de alimentos protetores foi categorizado em adequado (consumo $\geq 6$ vezes ao dia) e inadequado (consumo $<5$ vezes ao dia). O consumo de alimentos de risco ao excesso de gordura corporal foi classificado em adequado ( $\leq 2$ vezes ao dia) e inadequado (consumo $>3$ vezes ao dia). O número de refeições diárias dos escolares foi categorizado como variável dicotômica $\leq 3$ refeições/ dia e $>4$ refeições/dia. Realização do café da manhã foi categorizada em sim ou não ${ }^{32}$.

No questionário das atividades físicas, avaliado pelo Questionário de Atividade Física do dia Anterior (QUAFDA) $)^{31}, 11$ tipos de atividades físicas foram apresentadas (dançar, caminhar/ correr, pedalar, ajudar nas tarefas domésticas, subir escadas, jogar bola, pular corda, nadar, ginástica, andar de skate e brincar com o cachorro), com três intensidades distintas (devagar, rápido e muito rápido), o escolar foi orientado a assinalar as atividades físicas realizadas no dia anterior, bem como, escolher a intensidade que desempenhou por mais tempo. O nível geral de atividade física foi determinado ao somar os escores das atividades que o escolar referiu realizar. Foram atribuídos três pesos distintos como forma de ponderar as atividades assinaladas pelo escolar: peso um para atividades de intensidade leve (devagar), peso três para atividades de intensidade moderada (rápido) e peso nove para intensidade vigorosa (muito rápido) ${ }^{33}$. Na validação do QUAFDA, foram observados valores de sensibilidade e especificidade de $78 \%$ e $56 \%$, respectivamente ${ }^{33}$.

Os dados foram duplamente inseridos no programa Epi-Data ${ }^{\circledR}$ versão 3.2 (Epidata Association, Odense, Dinamarca) por digitadores previamente treinados. A análise estatística foi realizada no software STATA ${ }^{\circledR}$ (Statistical Software for Professionals, Texas), versão 11.0. As análises foram corrigidas pelo efeito do delineamento e plano amostral do estudo por meio da utilização do comando svy do Stata, tanto para as estimativas das prevalências de excesso de gordura corporal quanto para as análises das associações. Para a análise de associação, utilizou-se o teste de quiquadrado conforme variáveis independentes, sendo obtido o cálculo das prevalências e seus respectivos intervalos de confianças (IC95\%). Nas análises brutas e ajustadas foi utilizada a regressão de Poisson ${ }^{34}$ para a determinação das razões de prevalência e intervalos de confiança, uma vez que este procedimento mede a prevalência da condição de interesse no grupo de expostos em relação à prevalência no grupo dos não expostos, recurso estatístico utilizado em estudos epidemiológicos transversais quando a variável dependente apresenta prevalência acima de $20 \%^{34}$. Na análise ajustada foi seguido um modelo hierarquizado: no nível distal do desfecho foram consideradas as variáveis rede de ensino, sexo, renda familiar e escolaridade dos pais; e no nível proximal as variáveis consumo de alimentos protetores e de risco, realização do café da manhã, número de refeições e atividade física. Nesta seleção de variáveis por níveis mantiveram-se na análise aquelas com $\mathrm{p}<0,20$. Os resultados são apresentados pelas razões de prevalências e respectivos intervalos de confiança de $95 \%$, considerando o nível de significância estatístico para associação de $5 \%$.

O estudo foi aprovado pelo Comitê de Ética em Pesquisa com Seres Humanos da Universidade Federal de Santa Catarina conforme as normas estabelecidas pela Resolução 466/2012 do Conselho Nacional de Saúde.

\section{Resultados}

Foram coletados dados de 2506 escolares, entretanto, foram excluídos 25 alunos por falta de dados antropométricos completos, totalizando uma amostra final em 2.481 escolares. As características gerais da amostra investigada podem ser observadas na Tabela 1. O maior percentual dos escolares foi do sexo feminino (56,5\%); matriculados na rede pública (63,5\%); filhos de mães $(39,3 \%)$ e pais $(39,2)$ com escolaridade igual ou superior a 12 anos de estudo; pais com renda mensal familiar de $\mathrm{R} \$ 1.577,00$ a $\mathrm{R} \$$ $3.000,00(34,3 \%)$. As frequências de escolares que consumiam alimentos protetores e de risco 
de forma adequada foram de $8,4 \%$ e $40,5 \%$, respectivamente; sendo que $88,0 \%$ realizavam café da manhã; 99,0\% realizavam refeições diárias < 4 vezes por dia e $100 \%$ praticavam alguma atividade física.

Dos 2.481 escolares investigados, $23,9 \%$ foram classificados com excesso de gordura corporal (Tabela 1). O percentual foi maior nos meninos $25,8 \%$ em comparação as meninas $22,5 \%$, entretanto, não sendo estatisticamente significativo $(\mathrm{p}=0,359)$ (dados não apresentados em tabelas). O percentual de excesso de gordura corporal foi maior nos escolares de 11 a 14 anos de idade $(25,8 \%)$ em comparação aos de 7 a 10 anos de idade (22,8\%), não sendo estatisticamente significativo $(\mathrm{p}=0,202)$ (dados não apresentados em Tabelas).

A Tabela 2 apresenta as análises brutas e ajustadas dos escolares com idade de 7 a 10 anos. Tanto na análise bruta quanto na ajustada, nenhuma variável foi associada com a prevalência de excesso de gordura corporal.

A Tabela 3 apresenta as análises brutas e ajustadas dos escolares com idade de 11 a 14 anos. As variáveis que apresentaram valores significativos na análise bruta foram, consumo de alimento de risco $(p=0,011)$ e número de refeições $(p=$ $0,003)$. Na análise ajustada estas variáveis continuaram apresentando associação. Consumo de alimento de risco $(p=0,009)$, sendo observado que os escolares que consumiram mais de 3 vezes/dia estes alimentos apresentaram prevalência de excesso de gordura corporal 0,61 vezes menor que os que consumiram $\leq 2$ vezes/dia (IC95\%: 0,47-0,79). Os escolares que realizavam menos que 3 refeições/dia apresentaram prevalência de excesso de gordura corporal 1,62 vezes maior que os que realizavam $>4$ refeições/dia (IC95\%: 1,38-1,91).

\section{Discussão}

Em relação às limitações do estudo, é importante destacar a difícil tarefa observada na comparação da prevalência de excesso de gordura corporal com resultados de outros estudos, em virtude das diferentes faixas etárias, dos distintos métodos aplicados e dos pontos de corte adotados para o diagnóstico do excesso de gordura corporal nas pesquisas publicadas. Outra limitação deste estudo é o fato de que a equação para calcular o percentual de gordura não foi validada para crianças e adolescentes do Brasil, porém tais equações foram construídas com base em estimativas mais
Tabela 1. Caracterização da amostra investigada segundo as variáveis sociodemográficas, socioeconômicas e comportamentais em escolares de 7 a 14 anos de idade. Florianópolis, SC, Brasil, 2012/2013.

\begin{tabular}{|c|c|c|}
\hline Variáveis & $\mathbf{n}$ & $\%(\mathrm{IC} 95 \%)$ \\
\hline Total & 2506 & 100 \\
\hline \multicolumn{3}{|l|}{ Sexo do escolar $(n=2506)$} \\
\hline Masculino & 1172 & $43,5(40,1-46,9)$ \\
\hline Feminino & 1334 & $56,5(53,1-59,9)$ \\
\hline \multicolumn{3}{|l|}{ Rede de ensino $(\mathrm{n}=2506)$} \\
\hline Pública & 1637 & $63,5(63,4-67,2)$ \\
\hline Privada & 869 & $36,5(32,8-36,6)$ \\
\hline \multicolumn{3}{|l|}{ Escolaridade materna $(\mathrm{n}=2389)$} \\
\hline$\geq 12$ anos & 903 & $39,3(35,9-39,7)$ \\
\hline $9-11$ anos & 849 & $36,2(33,6-37,5)$ \\
\hline $0-8$ anos & 637 & $24,5(24,5-28,4)$ \\
\hline \multicolumn{3}{|l|}{ Escolaridade paterna $(n=2086)$} \\
\hline$\geq 12$ anos & 778 & $39,2(35,2-39,4)$ \\
\hline $9-11$ anos & 690 & $33,5(31,1-35,1)$ \\
\hline $0-8$ anos & 618 & $27,3(27,3-31,6)$ \\
\hline \multicolumn{3}{|l|}{ Renda mensal (reais) $(\mathrm{n}=2152)$} \\
\hline $1^{\circ}$ tercil $(<1576)$ & 718 & $31,9(31,3-35,4)$ \\
\hline $2^{\circ}$ tercil $(1577-3000)$ & 736 & $34,3(32,2-36,2)$ \\
\hline $3^{\circ}$ tercil $(>3001)$ & 698 & $33,8(30,5-34,4)$ \\
\hline \multicolumn{3}{|l|}{ Alimentos protetores $(\mathrm{n}=2506)$} \\
\hline Adequado ( $\geq 6$ vezes/dia) & 235 & $08,4(03,1-13,7)$ \\
\hline Inadequado (<5vezes/dia) & 2271 & $91,6(86,3-96,9)$ \\
\hline \multicolumn{3}{|l|}{ Alimentos de risco $(\mathrm{n}=2506)$} \\
\hline Adequado ( $\leq 2$ vezes/dia $)$ & 1086 & $40,5(28,2-52,7)$ \\
\hline Inadequado (> 3 vezes/dia) & 1420 & $59,5(47,3-71,8)$ \\
\hline \multicolumn{3}{|l|}{ Café da manhã $(\mathrm{n}=2506)$} \\
\hline Sim & 2198 & $88,0(85,0-90,9)$ \\
\hline Não & 308 & $12,0(09,1-14,9)$ \\
\hline \multicolumn{3}{|l|}{$\begin{array}{l}\text { Número de refeições/dia }(\mathrm{n}= \\
2506)\end{array}$} \\
\hline$>4$ refeições & 2477 & $99,0(98,6-99,5)$ \\
\hline$\leq 3$ refeições & 29 & $01,0(00,5-01,4)$ \\
\hline Atividade física $(\mathrm{n}=2468)$ & 2468 & 100 \\
\hline \multicolumn{3}{|l|}{$\%$ GC $(\mathrm{n}=2481)$} \\
\hline Sem excesso de GC & 1890 & $76,1(67,0-85,2)$ \\
\hline Com excesso de GC ${ }^{a}$ & 591 & $23,9(14,8-33,0)$ \\
\hline
\end{tabular}

${ }^{a}$ Com excesso de GC: $\geq 25$ para meninos e $\geq 30$ para meninas; \%: Prevalência; \%GC: Percentual de Gordura Corporal; IMC (Índice de Massa Corporal).

precisas da composição corporal como a densidade, a água corporal e o conteúdo mineral ósseo $^{26}$.

Destaca-se o uso de uma amostra representativa de escolares da cidade de Florianópolis, seguindo um rigor metodológico por meio do treinamento prévio da equipe, com dados antropométricos aferidos diretamente da população investigada, utilização de instrumentos validados 
Tabela 2. Análises bruta e ajustada da associação entre gordura corporal e variáveis independentes nos escolares de 7 a 10 anos de idade. Florianópolis, SC, Brasil, 2012/2013.

\begin{tabular}{|c|c|c|c|c|}
\hline Variáveis & $\mathbf{n}$ & $\begin{array}{c}\text { Com excesso } \\
\text { de GC } \\
\%(\text { IC } 95 \%)\end{array}$ & $\begin{array}{c}\text { Análise } \\
\text { Bruta } \\
\text { RP (IC95\%) }\end{array}$ & $\begin{array}{c}\text { Análise } \\
\text { Ajustada } \\
\text { RP (IC95\%) }\end{array}$ \\
\hline Total & 1520 & $37,8(32,2-43,5)$ & - & - \\
\hline Sexo & & 0,275 & 0,274 & 0,343 \\
\hline Masculino & 722 & $26,2(11,9-40,5)$ & 1,00 & 1,00 \\
\hline Feminino & 798 & $20,0(09,4-30,6)$ & $0,76(0,39-1,45)$ & $0,74(0,31-1,72)$ \\
\hline Rede de ensino & & 0,075 & 0,072 & 0,074 \\
\hline Pública & 994 & $19,5(19,0-24,1)$ & 1,00 & 1,00 \\
\hline Privada & 526 & $27,8(21,2-28,6)$ & $1,42(0,94-2,14)$ & $1,39(0,94-2,06)$ \\
\hline Escolaridade materna & & 0,058 & 0,325 & 0,279 \\
\hline$\geq 12$ anos & 564 & $25,4(15,7-35,0)$ & 1,00 & 1,00 \\
\hline $9-11$ anos & 531 & $20,1(11,8-28,4)$ & $0,79(0,71-0,88)$ & $0,96(0,57-1,60)$ \\
\hline $0-8$ anos & 371 & $23,3(16,3-30,4)$ & $0,92(0,72-1,16)$ & $1,23(0,74-2,04)$ \\
\hline Escolaridade paterna & & 0,456 & 0,830 & 0,765 \\
\hline $0-8$ anos & 489 & $24,5(11,8-37,3)$ & 1,00 & 1,00 \\
\hline $9-11$ anos & 439 & $20,2(11,1-29,2)$ & $0,82(0,47-1,40)$ & $0,90(0,33-2,46)$ \\
\hline$\geq 12$ anos & 345 & $24,3(19,2-29,4)$ & $0,99(0,58-1,66)$ & $1,13(0,49-2,58)$ \\
\hline Renda mensal (reais) & & 0,490 & 0,363 & 0,900 \\
\hline $1^{\circ}$ tercil $(<1576)$ & 433 & $20,3(10,7-29,9)$ & 1,00 & 1,00 \\
\hline $2^{\circ}$ tercil $(1577-3000)$ & 469 & $22,6(16,2-28,9)$ & $1,11(0,58-2,13)$ & $1,02(0,51-2,02)$ \\
\hline $3^{\circ}$ tercil $(>3001)$ & 412 & $25,5(10,1-41,0)$ & $1,26(0,62-2,54)$ & $0,92(0,43-1,98)$ \\
\hline Alimentos protetores & & 0,882 & 0,882 & 0,802 \\
\hline Adequado ( $\geq 6$ vezes/dia) & 162 & $23,3(04,6-42,0)$ & 1,00 & 1,00 \\
\hline Inadequado (< 5vezes/dia) & 1358 & $22,7(14,1-31,4)$ & $0,97(0,60-1,57)$ & $1,05(0,61-1,78)$ \\
\hline Alimentos de risco & & 0,146 & 0,134 & 0,204 \\
\hline Adequado ( $\leq 2$ vezes/dia) & 628 & $26,3(12,1-40,5)$ & 1,00 & 1,00 \\
\hline Inadequado ( $>3$ vezes/dia) & 892 & $20,6(13,7-27,5)$ & $0,78(0,53-1,14)$ & $0,80(0,52-1,23)$ \\
\hline Café da manhã & & 0,149 & 0,118 & 0,362 \\
\hline Sim & 1376 & $22,1(13,8-30,4)$ & 1,00 & 1,00 \\
\hline Não & 144 & $29,6(06,5-52,7)$ & $1,34(0,87-2,06)$ & $1,18(0,75-1,87)$ \\
\hline Número de refeições/dia & & 0,155 & 0,113 & 0,289 \\
\hline$>4$ refeições & 1504 & $22,6(13,2-32,0)$ & 1,00 & 1,00 \\
\hline$\leq 3$ refeições & 16 & $43,9(05,7-56,8)$ & $1,94(0,74-5,04)$ & $1,53(0,55-4,24)$ \\
\hline \multirow[t]{2}{*}{ Atividade física } & & 0,735 & 0,716 & 0,635 \\
\hline & 1504 & $13(7-22)^{\mathrm{a}}$ & $1,00(0,98-1,02)$ & $1,00(0,98-1,02)$ \\
\hline
\end{tabular}

${ }^{a}$ Mediana e Intervalo Interquartil; GC: Gordura corporal; \%: Prevalência; IC95\%: Intervalo de 95\% de confiança; RP: Razão de Prevalência.

para coleta de dados e a dupla digitação de dados para evitar vieses de compilação, o que confere confiabilidade aos resultados deste estudo.

A prevalência de excesso de gordura corporal encontrada no estudo é preocupante, uma vez que pode continuar na vida adulta e causar consequências como diabetes melittus tipo 2, hipertensão arterial, dislipidemias, síndrome metabólica e doença arterial coronariana ${ }^{35}$.

Comparando as prevalências de excesso de gordura corporal, conferidas pelas DCT e DCS, valores maiores foram observados em Santa Ca- tarina, em adolescentes de 14 a 17 anos de idade $(42,3 \% \text { com excesso de gordura corporal })^{20}$, enquanto que valores parecidos foram encontrados em Minas Gerais com escolares de 7 a 9 anos de idade, em que se observou excesso de gordura corporal nos meninos de $18,2 \%$ e nas meninas de $23,9 \%{ }^{21}$.

Estudos internacionais apresentaram percentual de gordura corporal inferiores, como no Peru, em escolares de 6 a 10 anos de idade a prevalência nos meninos foi de $14,3 \%$ e nas meninas de $25,0 \%{ }^{36}$. Em Portugal, em adolescentes de 11 a 
Tabela 3. Análises bruta e ajustada da associação entre gordura corporal e variáveis independentes nos escolares de 11 a 14 anos de idade. Florianópolis, SC, Brasil, 2012/2013.

\begin{tabular}{|c|c|c|c|c|}
\hline Variáveis & $\mathbf{n}$ & $\begin{array}{c}\text { Com excesso } \\
\text { de GC } \\
\%(\text { IC } 95 \%)\end{array}$ & $\begin{array}{c}\text { Análise } \\
\text { Bruta } \\
\text { RP (IC95\%) }\end{array}$ & $\begin{array}{c}\text { Análise } \\
\text { Ajustada } \\
\text { RP (IC95\%) }\end{array}$ \\
\hline Total & 961 & $46,7(42,1-51,2)$ & - & - \\
\hline Sexo & & 0,781 & 0,784 & 0,716 \\
\hline Masculino & 438 & $24,8(05,7-43,9)$ & 1,00 & 1,00 \\
\hline Feminino & 523 & $26,4(20,8-32,0)$ & $1,06(0,54-2,08)$ & $1,08(0,56-2,07)$ \\
\hline Rede de ensino & & 0,664 & 0,659 & 0,805 \\
\hline Pública & 624 & $24,6(22,0-28,9)$ & 1,00 & 1,00 \\
\hline Privada & 337 & $28,2(21,1-30,5)$ & $1,14(0,47-2,75)$ & $1,13(0,45-2,86)$ \\
\hline Escolaridade materna & & 0,618 & 0,809 & 0,636 \\
\hline$\geq 12$ anos & 334 & $23,8(02,4-45,4)$ & 1,00 & 1,00 \\
\hline $9-11$ anos & 311 & $29,3(16,9-41,8)$ & $1,22(0,51-2,94)$ & $1,38(0,86-2,21)$ \\
\hline $0-8$ anos & 255 & $43,2(20,5-29,5)$ & $1,05(0,46-2,37)$ & $1,29(0,45-3,73)$ \\
\hline Escolaridade paterna & & 0,158 & 0,603 & 0,889 \\
\hline $0-8$ anos & 286 & $23,6(07,5-39,6)$ & 1,00 & 1,00 \\
\hline $9-11$ anos & 243 & $32,5(17,0-48,0)$ & $1,37(0,88-2,14)$ & $1,62(0,90-2,90)$ \\
\hline$\geq 12$ anos & 260 & $26,3(21,5-31,1)$ & $1,11(0,62-1,98)$ & $1,19(0,65-2,18)$ \\
\hline Renda mensal (reais) & & 0,598 & 0,691 & 0,902 \\
\hline $1^{\circ}$ tercil $(<1576)$ & 275 & $25,4(15,5-35,3)$ & 1,00 & 1,00 \\
\hline $2^{\circ}$ tercil $(1577-3000)$ & 258 & $22,3(04,4-40,2)$ & $0,87(0,50-1,53)$ & $0,77(0,31-1,88)$ \\
\hline $3^{\circ}$ tercil $(>3001)$ & 282 & $27,9(16,3-39,5)$ & $1,09(0,55-2,19)$ & $1,10(0,70-1,73)$ \\
\hline Alimentos protetores & & 0,200 & 0,190 & 0,225 \\
\hline Adequado ( $\geq 6$ vezes/dia) & 71 & $35,1(15,2-55,0)$ & 1,00 & 1,00 \\
\hline Inadequado (< 5vezes/dia) & 890 & $25,2(15,0-35,4)$ & $0,71(0,38-1,34)$ & $0,74(0,39-1,38)$ \\
\hline Alimentos de risco & & 0,012 & 0,011 & 0,009 \\
\hline Adequado ( $\leq 2$ vezes/dia) & 443 & $33,7(21,1-46,3)$ & 1,00 & 1,00 \\
\hline Inadequado ( $>3$ vezes/dia) & 518 & $19,8(13,1-26,5)$ & $0,58(0,43-0,78)$ & $0,61(0,47-0,79)$ \\
\hline Café da manhã & & 0,077 & 0,063 & 0,120 \\
\hline $\operatorname{Sim}$ & 806 & $23,5(13,8-33,3)$ & 1,00 & 1,00 \\
\hline Não & 155 & $37,0(14,6-59,4)$ & $1,57(0,95-2,59)$ & $1,43(0,84-0,79)$ \\
\hline Número de refeições/dia & & 0,010 & 0,003 & 0,002 \\
\hline$>4$ refeições & 951 & $25,4(15,5-35,3)$ & 1,00 & 1,00 \\
\hline$\leq 3$ refeições & 10 & $66,3(22,4-97,0)$ & $2,61(1,85-3,68)$ & $1,62(1,38-1,91)$ \\
\hline \multirow[t]{2}{*}{ Atividade física } & & 0,666 & 0,665 & 0,705 \\
\hline & 964 & $12(6-21)^{a}$ & $1,00(0,97-1,03)$ & $1,00(0,97-1,03)$ \\
\hline
\end{tabular}

${ }^{a}$ Mediana e Intervalo Interquartil; GC; Gordura corporal; \%: Prevalência; IC95\%: Intervalo de 95\% de confiança; RP: Razão de Prevalência.

15 anos de idade, o percentual de gordura corporal foi de $26,5 \%{ }^{37}$.

Quanto aos fatores associados ao excesso de gordura corporal, os resultados deste estudo confirmam a influência de hábitos comportamentais destes escolares, os quais podem contribuir para o aumento desta prevalência, com particularidades específicas e significativas para cada idade investigada.

Em relação à rede de ensino, o estudo não identificou associação significativa com a prevalência de excesso de gordura corporal nos es- colares. Resultado oposto observado por Ramos et al. ${ }^{17}$, os quais identificaram que os alunos das escolas privadas apresentaram uma prevalência 1,3 vezes maior de percentual alto de gordura corporal. Ressalta-se que condições econômicas elevadas podem levar a um consumo exagerado de alimentos e mais acesso às compras de alimentos calóricos, enquanto que nas classes menos favorecidas a quantidade destes alimentos para o consumo é menor ${ }^{17,38}$. Outro fator a ser considerado é que o nível econômico mais elevado pode influenciar na redução da atividade física destes 
escolares, que são substituídas por computadores e jogos eletrônicos, além da falta de tempo dos pais, impedindo que as crianças brinquem em parques, praças e ruas ${ }^{17}$.

No presente estudo, observou-se que a escolaridade dos pais não apresentou associação significativa com a prevalência de excesso de gordura corporal nos escolares. No estudo realizado por Coelho et al..$^{22}$ em Minas Gerais, com escolares de 6 a 14 anos de idade, também não foi possível observar associações significativas. Cabe ressaltar que a educação materna pode contribuir para o conhecimento de práticas saudáveis e não saudáveis, as quais consequentemente podem interferir no peso corporal da criança ${ }^{15}$.

Em relação à renda mensal familiar, o estudo não apresentou significância com a prevalência de excesso de gordura corporal nos escolares. No estudo realizado por Coelho et al..$^{22}$, não houve associação entre a gordura corporal dos escolares e renda familiar. Porém, no estudo realizado por Duquia et al. ${ }^{14}$ em Pelotas, avaliando as dobras tricipital e subescapular, os adolescentes de pais com melhores condições socioeconômicas, tinham maior percentual de gordura corporal, apresentando associação significativa com a renda familiar.

Em relação ao consumo de alimentos de risco, pode-se observar que um pouco mais da metade dos escolares $(59,5 \%)$ os consomem de forma inadequada ( $>$ 3vezes/dia). Entre os escolares de 11 a 14 anos de idade, foi observado que quem consumia $>3$ vezes/dia tinha menores prevalências de excesso de gordura corporal. $\mathrm{O}$ fato de o consumo de alimentos de risco mostrar efeito inverso sobre o excesso de gordura corporal expressa um resultado contrário ao que a literatura tem apresentado, sendo que escolares que consomem mais estes alimentos têm risco aumentado para excesso de gordura corporal, exigindo assim a realização de novos estudos que busquem o aprofundamento de tais achados. Em geral, se atribui que os adolescentes parecem não dar importância para as consequências dos hábitos alimentares inadequados à saúde, apresentando maior preferência ao consumo de guloseimas, lanches e refrigerantes ${ }^{39}$.

Duquia et al. ${ }^{14}$, avaliaram 4482 escolares de 11 anos de idade no Rio Grande Sul e ao comparar a dieta inadequada, baixo consumo de fibras e alto consumo de gordura com as dobras cutâneas tricipital e subescapular, também não encontraram associação. Por outro lado, observa-se que há estudos que encontraram associação entre consumo alimentar e sobrepeso/obesidade em escolares $^{10-12}$, reforçando assim a importância de mais pesquisas para verificar a associação desta variável e excesso de gordura corporal.

Ressalta-se que os alimentos do grupo das frutas, legumes e verduras são considerados mais saudáveis e contribuem para a proteção de doenças crônicas não transmissíveis (DCNT), entre elas a obesidade. Por outro lado, os alimentos dos grupos dos açúcares, gorduras e o sal são considerados de risco para as DCNT, devendo ter o consumo reduzido ${ }^{32}$. Por isso, a importância da realização de atividades para a promoção de hábitos alimentares mais saudáveis para os escolares, a fim de prevenir o aumento da gordura corporal e consequentemente o surgimento de $\mathrm{DCNT}^{10-12}$.

Nos escolares avaliados, o café da manhã não apresentou associação com o excesso de gordura corporal. Segundo estudo realizado por Ghosh ${ }^{12}$, na Îndia, com 1.061 escolares de 8 a 18 anos de idade, verificou-se que os escolares com hábitos de pular a refeição do café da manhã são mais obesos, porém neste estudo a obesidade foi avaliada pelo índice de massa corporal (IMC). Uma possível explicação da importância da realização do café da manhã, é que crianças que fariam essa refeição teriam maior consumo de grãos, frutas e produtos lácteos ${ }^{13}$. Ressalta-se que o consumo frequente e adequado do café da manhã pode melhorar o poder de saciedade e assim, limitar o consumo de lanches calóricos por crianças e adolescentes ao longo do dia. Além do controle do peso, outro fator importante é que o café da manhã ajuda no rendimento escolar dos estudantes, influenciando positivamente na concentração e memória para as atividades escolares ${ }^{40}$.

Em relação ao número de refeições, apresentou associação significativa com o excesso de gordura corporal em escolares de 11 a 14 anos. Em um estudo realizado por Eloranta et al. ${ }^{11}$ com escolares de 6 a 8 anos de idade na Finlândia, foi observado que aqueles que consumiam as 3 principais refeições, apresentavam um menor percentual de gordura corporal (cabe ressaltar que neste estudo foi utilizada a bioimpedância). Evidências apontam que o ato de comer de forma irregular ou pular refeições diminui a sensação de saciedade e, consequentemente, faz com que aumente o consumo de alimentos nas próximas refeições. ${ }^{11}$ Sendo assim, recomenda-se a realização de pelo menos três refeições diárias (café da manhã, almoço e jantar), intercaladas com pequenos lanches ${ }^{32}$, como estratégia para constituição de hábitos alimentares saudáveis e, por consequência, diminuição do risco de acúmulo de gordura corporal. 
Quanto à prática de atividade física o estudo não apresentou associação significativa com excesso de gordura corporal. No estudo realizado por Brito et al. ${ }^{16}$, com estudantes de 13 a 16 anos de idade em Teresina, Piauí, observou-se que o nível de atividade física alto estava associado a menor percentual de gordura corporal. Por sua vez, Coelho et al. ${ }^{22}$, em Minas Gerais, com escolares de 6 a 14 anos de idade, não identificaram diferença significativa entre os escolares ativos com o percentual de gordura corporal, resultado avaliado através de bioimpedância. Em um estudo realizado por Vasconcellos et al. ${ }^{7}$ em Niterói, com 328 escolares de 10 a 18 anos de idade, não foi identificada associação com atividade física e excesso de peso, porém os autores concluíram que o tempo de tela, mais que $28 \mathrm{~h} /$ semana, associou-se significativamente ao excesso de peso (avaliado pelo IMC), constatando assim, que há um estilo de vida inadequado nos adolescentes. É importante ressaltar que a escola é um ambiente adequado para a estimulação de um comportamento mais ativo em crianças e adolescentes. É um local relativamente seguro, há mais disponibilidade de equipamentos e espaços, além de oferecer uma orientação profissional mais adequada $^{41}$.

Por fim, conclui-se neste estudo que o percentual de gordura corporal associou-se a diferentes fatores da população investigada como número de refeições (consumir menos que 3 refeições ao dia) e alimento de risco (consumir mais que 3 vezes ao dia). Convém destacar, que a família exerce importante influência sobre o modo de vida das crianças e adolescentes. Orientações a respeito de hábitos de vida saudáveis (incluindo alimentação e atividade física) são ações essenciais que devem ser estimuladas nos contextos familiar e escolar, como forma de contribuir para a possível modificação de hábitos inadequados e, consequentemente, contribuir com a melhoria das condições de saúde e nutrição das crianças e adolescentes.

\section{Colaboradores}

GL D’Ávila, DAS Silva e FAG Vasconcelos participaram da concepção do trabalho, da análise e interpretação dos dados e de sua redação ou revisão crítica. Todos os autores leram e aprovaram a versão final. 


\section{Referências}

1. Li P Yang F, Xiong F, Huo T, Tong Y, Yang S, Mao M. Nutritional status and risk factors of overweight and obesity for children aged 9-15years in Chengdu, Southwest China. BMC Public Health 2012; 12:636.

2. Levy TS, Nasu LC, Humaran IMG, Aguilar AJ, Ramirez AJM, Villalpando S. La obesidad en ninos mexicanos en edad escolar se asocial con el consumo de alimentos fuera del hogar: durante el trayecto de la casa a la escuela. ALAN 2011; 61(3):288-295.

3. Mushtaq MU, Gull S, Mushtaq K, Shahid U, Shad MA, Akram J. Dietary behaviors, physical activity and sedentary lifestyle associated with overweight and obesity, and their socio-demographic correlates, among Pakistani primary school children. Int J Behav Nutr Phys Act 2011; 8:130.

4. O'Dea J, Amy N. Perceived and desired weight, weight related eating and exercising behaviours, and advice received from parents among thin, overweight, obese or normal weight Australian children and adolescents. Int J Behav Nutr Phys Act 2011; 8(1):68.

5. Pelegrini A, Silva DAS, Petroski EL, Gaya ACA. Sobrepeso e obesidade em escolares brasileiros de sete a nove anos: dados do projeto Esporte Brasil. Rev Paul Pediatr 2010; 28(3):290-295.

6. Ribas SA, Silva LCS. Fatores de risco cardiovascular e fatores associados em escolares do Município de Belém, Pará, Brasil. Cad Saude Publica 2014; 30(3):577-586.

7. Vasconcellos MB, Anjos LA, Vasconcellos MTL. Estado nutricional e tempo de tela de escolares da Rede Pública de Ensino Fundamental de Niterói, Rio de Janeiro, Brasil. Cad Saude Publica 2013; 29(4):713-722.

8. Assis MAA, Rolland-Cachera MF, Grosseman S, Vasconcelos FA, Luna ME, Calvo MC, Barros MV, Pires MM, Bellisle F. Obesity, overweight and thinness in schoolchildren of the city of Florianopolis, Southern Brazil. Eur J Clin Nutr 2005; 59(9):1015-1021.

9. Schmitz BAS, Corso ACT, Caldeira GV, Gimeno SGA, Gabriel CG, Vasconcelos FAG. Overweight and obesity related factors in schoolchildren in Santa Catarina State, Brazil. Arch Latinoam Nutr 2010; 60(4):332-339.

10. Amin T, Al-Sultan A, Ali A. Overweight and obesity and their relation to dietary habits and socio-demographic characteristics among male primary school children in Al-Hassa, Kingdom of Saudi Arabia. Eur J Nutr 2008; 47(6):310-318.

11. Eloranta AM, Lindi V, Schwab U, Tompuri T, Kiiskinen S, Lakka HM, Laitinen T, Lakka TA. Dietary factors associated with overweight and body adiposity in Finnish children aged 6-8 years: the PANIC Study. Int J Obes 2012; 36(7):950-955.
12. Ghosh A. Explaining overweight and obesity in children and adolescents of Asian Indian origin: the Calcutta childhood obesity study. Indian J Public Health 2014; 58(2):125-128.

13. Nicklas TA, Baranowski T, Cullen KW, Berenson G. Eating patterns, dietary quality and obesity. J Am Coll Nutr 2001; 20(6):599-608.

14. Duquia RP, Dumith SC, Reichert FF, Madruga SW, Duro LN, Menezes AMB, Araújo CL. Epidemiologia das pregas cutâneas tricipital e subescapular elevadas em adolescentes. Cad Saude Publica 2008; 24(1):113-121.

15. Keane E, Layte R, Harrington J, Kearney PM, Perry IJ. Measured Parental Weight Status and Familial Socio-Economic Status Correlates with Childhood Overweight and Obesity at Age 9. PLoS One 2012; 7:8.

16. Brito AKA, Silva Junior FL, Silva CL, Franca NM. Nível de atividade física e correlação com o índice de massa corporal e percentual de gordura em adolescentes escolares da cidade de Teresina-PI. Rev Bras Ativ Fís Saúde 2012; 17(3):212-216.

17. Ramos MLM, Pontes ERJC, Ramos MLM, Barros VRSP. Sobrepeso e obesidade em escolares de 10 a 14 anos. Rev Bras Promoc Saúde 2013; 26(2):223-232.

18. Moreno LA, Moliner-Urdiales D, Ruiz JR, Mesana MI, Vicente-Rodriguez G, Rodriguez G, Fleta J, León JF, García-Fuentes M, Castillo MJ, González-Gross M, Marcos A; AVENA study group; HELENA study group. Five year trends on total and abdominal adiposity in Spanish adolescents. Nutr Hosp 2012; 27(3):731-738.

19. Gomes FS, Anjos LA, Vasconcelos MTL. Antropometria como ferramenta de avaliação do estado nutricional coletivo de adolescentes: uma revisão da literatura. Revista de Nutrição 2010; 23(4):591-605.

20. Silva DA, Berria J, Grigollo LR, Petroski EL. Prevalence and factors associated with high body fat in adolescents from a region of Brazil. J Community Health 2012; 37(4):791-798.

21. Barbosa L, Chaves OC, Ribeiro RCL. Parâmetros antropométricos e de composição corporal na predição do percentual de gordura e perfil lipídico em escolares. Rev Paul Pediatr 2012; 30(4):520-528.

22. Coelho LG, Candido APC, Machado-Coelho GLL, Freitas SN. Associação entre estado nutricional, hábitos alimentares e nível de atividade física em escolares. $J$ Pediatr 2012; 88(5):406-412.

23. Motter AF, Vasconcelos FAG, Correa EM, Andrade DF. Pontos de venda de alimentos e associação com sobrepeso/obesidade em escolares de Florianópolis, Santa Catarina, Brasil. Cad Saude Publica 2015; 31(3):620632. 
24. Bernardo CO, Fernandes PS, Campos RMMB, Adami F, Vasconcelos FAG. Associação entre o índice de massa corporal de pais e de escolares de 7 a 14 anos de Florianópolis, SC, Brasil. Rev Bras Saúde Mater Infant 2010; 10(2):183-190.

25. Habicht JP. Standardization of quantitative epidemiological methods in the field. Bolletin de la Oficina Sanitaria Panamericana 1974; 76(5):375-384.

26. Lohman TG. Applicability of body composition techniques and constants for children and youths. In: Pandolf KB, editor. Exercise and Sport Sciences Reviews. New York: Macmillan; 1986. p. 325-357.

27. Pires-Neto CS, Petroski EL. Assuntos sobre as equações da gordura corporal relacionadas às crianças e jovens. In: Carvalho S, organizador. Comunicação, Movimento e Mídia na educação Física. Santa Maria: Imprensa Universitária, UFSM; 1996. p. 21-30.

28. Lohman TG, Roche AFE, Martorell R. Anthropometric Standardization Reference Manual. Champign: Human Kinetics Books; 1991.

29. Williams DP, Going SB, Lohman TG, Harsha DW, Srinivasan SR, Webber LS, Berenson GS. Body fatness and risk for elevated blood pressure, total cholesterol, and serum lipoprotein ratios in children and adolescents. Am J Public Health 1992; 82(3):358-363.

30. Assis MAA, Benedet J, Kerpel R, Vasconcelos FAG, Di Pietro PF, Kupek E. Validação da terceira versão do Questionário Alimentar do Dia Anterior (QUADA-3) para escolares de 6 a 11 anos. Cad Saude Publica 2009; 25(8):1816-1826.

31. Cabral LGA, Costa FF, Liparotti JR. Evidencias preliminares de validade da seção de atividade física do Questionário de Atividade Física e Alimentação do Dia Anterior (QUAFDA). Rev Bras Ativ Fís Saúde 2011; 16(2):100-106.

32. Brasil. Ministério da Saúde (MS). Secretaria de Atenção a Saúde. Departamento de Atenção Básica. Coordenação Geral da Política de Alimentação e Nutrição. Guia alimentar para a população brasileira: promovendo a alimentação saudável. Brasília: MS; 2008.

33. Costa FF, Assis MAA. Nível de atividade física e comportamentos sedentários de escolares de sete a dez anos de Florianópolis-SC. Rev Bras Ativ Fís Saúde 2011; 16(1):48-54.

34. Barros AJ, Hirakata VN. Alternatives for logistic regression in cross-sectional studies: an empirical comparison of models that directly estimate the prevalence ratio. BMC Med Res Methodol 2003; 3:21.
35. Gupta N, Shah P, Nayyar S, Misra A. Childhood obesity and the metabolic syndrome in developing countries. Indian J Pediatr 2013; 80(Supl. 1):S28-S37.

36. Cossio-Bolanos MA, Pablos AC, Arruda M. Assessment of body adiposity in school students in Arequipa, Peru. Rev Peru Med Exp Salud Publica 2012; 29(4):477-482.

37. Coelho-e-Silva MJ, Vaz Ronque ER, Cyrino ES, Fernandes RA, Valente-dos-Santos J, Machado-Rodrigues A, Martins R, Figueiredo AJ, Santos R, Malina RM. Nutritional status, biological maturation and cardiorespiratory fitness in Azorean youth aged 11-15 years. BMC Public Health 2013; 13:495.

38. Johnson CA, Xie B, Liu C, Reynolds KD, Chou CP, Koprowski C, Gallaher P, Spruitj-Metz D, Guo Q, Sun P, Gong J, Palmer P. Sociodemographic and cultural comparison of overweight and obesity risk and prevalence in adolescents in Southern California and Wuhan, China. J Adolesc Health 2006; 39(6):925-928.

39. Ochsenhofer K, Quintella LCM, Silva EC, Nascimento APB, Ruga GMNA, Philippi ST, Szarfac SC. The school's role in making-up food choice: school feeding or junk food? Nutrire: Nutrire Sao Paulo 2006; 31(1):116.

40. Trancoso SC, Cavalli SB, Proença RPC. Café da manhã: caracterização, consumo e importância para a saúde. Rev. Nutr 2010; 23(5):859-869.

41. Rezende LFM, Azeredo CM, Canella DS, Claro RM, Castro IR, Levy RB, Luiz OC. Sociodemographic and behavioral factors associated with physical activity in Brazilian adolescents. BMC Public Health 2014; 14:485.

Artigo apresentado em 02/03/2015

Aprovado em 11/09/2015

Versão final apresentada em 13/09/2015 
